\title{
An attempt to identify the interanimal retardation factor in the kindling effect by thin layer chromatography
}

\author{
JOHN GAITO \\ York University, Downsview, Ontario, Canada MSJ 1PS
}

\begin{abstract}
Various solvents and chromatographic plates were used in thin layer chromatography in an attempt to determine unique patterns resulting during the kindling process. The patterns were the same for nonstimulated controls and for rats at various points of behavioral changes.
\end{abstract}

The kindling paradigm (Goddard, McIntyre, \& Leech, 1969) was instituted in our laboratory a few years ago to determine brain chemical events which accompany the behavioral changes. In one set of experiments, the "kindling effect" was paired with the "transfer experiment" (Gaito, 1971). These experiments involved the evaluation of the effects of brain homogenate supernatant from kindled (E) and nonkindled (C) rats on the kindling rates of recipients. The results showed that a "retardation effect" was present when rats were injected intraperitoneally (i.p.) with supernatant from kindled rats, if the amount rejected was equivalent to that from one or more brains (Gaito \& Gaito, 1974; Gaito, 1975, 1976). Other research concerned with the isolation and identification of the molecular species producing the negative transfer suggested that the active component had a molecular weight greater than 500 but less than 10,000 (Gaito, 1975, 1976).

As these experiments were progressing, a number of other experiments were underway attempting to determine chemical changes during the kindling process. In a previous report, a comparison of acidic proteins using polyacrylamide gel electrophoresis found no quantitative or qualitative differences in gel patterns between rats at any stage in the kindling process (Gaito, Hopkins, \& Pelletier, 1973). In this paper, we report the results of experiments using thin layer chromatography (TLC) to evaluate patterns of amino acid constituents for rats at various points in the kindling series. TLC has been used successfully by Ungar, Desiderio, and Parr (1972) to identify a unique polypeptide which they report is responsible for avoidance behavior in passive avoidance conditioning. We were

Research reported in this paper was supported by the President's NRC Fund (Grant 32) from York University and a grant from the Ontario Society for Crippled Children. I wish to thank A. E. Eisenstark (Chairman, Division of Biological Sciences) and S. Brown (Chairman, Department of Psychology) for their generosity in allowing me the use of their facilities and animals at the University of Missouri, and F. Landau, B. Landau, and J. McCormick for guidance during the initial chromatography work. attempting to determine if there is a unique polypeptide produced during the development of convulsions. Based on the results of our previous work, we expected a qualitatively different TLC pattern for E rats than for C animals, especially in a fraction varying in molecular weight between 500 and 10,000. However, no difference in patterns between $\mathrm{E}$ and $\mathrm{C}$ were detected.

\section{METHOD AND RESULTS}

In all experiments, experimental and control rats (E and $C$ ) had electrodes implated unilaterally in the amygdala. Six or more days later, the kindling procedures began. Experimental rats were stimulated three times daily (with $1 \mathrm{~h}$ between trials) by a $60-\mathrm{Hz}$ sine wave of $100-\mu \mathrm{A}$ intensity (peak to peak) for $30 \mathrm{sec}$ from a Lafayette sine wave stimulator. At a specific point in the kindling trials or at a specific clonic convulsion (CC) trial, at $10 \mathrm{~min}$ after the initiation of the trial, the rats were sacrificed by decapitation. The brain was rapidly removed; the olfactory bulbs, cerebellum, and brain stem parts below the cerebellum were deleted; the remainder was used for injections. Control donors (C) were connected to stimulator lead wires but received no stimulation. The $\mathrm{Cs}$ were sacrificed and treated in the same manner as the Es.

The $\mathrm{E}$ brains were homogenized together in physiological saline or in distilled water. A similar procedure was used with $\mathrm{C}$ brains. The homogenates were shaken over night at $2^{\circ} \mathrm{C}$ and centrifuged at $20,000 \mathrm{x}$ g for $1 \mathrm{~h}$. The homogenate supernatants from each of the $E$ and C rats were polled separately and used for analyses. In Experiments I and II, conducted at the University of Missouri, Sprague-Dawley rats were used. Wistar rats were those employed for research at York University (Experiments III, IV, and V). Rats were approximately 100 days of age when electrodes were implanted.

The major portion of the chromatography work attempted was ascending one-dimensional TLC. A few runs with two-dimensional TLC were attempted also in Experiment III. 


\section{Experiment I: Exploratory}

Prior to initiation of formal experiments with TLC procedures, it was necessary to determine the specific type of chromatogram and specific solvent which would provide the best resolution of sample spots. Two types of plates (cellulose, silica gel) and three solvent systems (Solvent 1: $\mathrm{n}$ butanol-ethanol-acetic acid-water, 8:2:1:3; Solvent 2: $\mathrm{n}$ butanol-pyridineacetic acid-water, 5:3.5:1:4; Solvent 3 , $\mathrm{n}$ butanolpyridine-water, $1: 1: 1)$ were evaluated. The best resolution was achieved with a cellulose chromatographic plate in Solvent 1 . It was found also that the clearest pattern was provided if the sample was dissolved in distilled water rather than in physiological saline for both plates and with all solvent systems except Solvent 1 . In this solvent, the patterns were basically the same with samples in distilled water or in saline, although Spots 3 and 4 tended to fuse more with saline than with water (Figure 1A). However, because previous studies involving the retardation effect used saline for the samples, physiological saline was employed for Experiments II to V.

\section{Experiment II}

Twelve Sprague-Dawley rats were brought to specific points in the kindling process prior to sacrificing. There were four groups of three rats each: control, nonstimulated (C); stimulated to behavioral automatism stage $\left(E_{1}\right)$; stimulated to $4 \mathrm{CC}\left(\mathrm{E}_{2}\right)$; stimulated to $13 \mathrm{CC}\left(\mathrm{E}_{3}\right)$.

Samples of the supernatant from each of the four groups were spotted on a cellulose plate and run in Solvent 1. There were three separate runs. All four samples provided the same pattern after spraying with ninhydrin reagent, 10 spots, those shown in Figure 1A. The average $R f$ values, indicating relative rate of migration (distance from origin for sample/distance from origin for solvent front), for each of the 10 were .08 , $.16, .22, .25, .35, .42, .48, .54, .60$, and .84 .

\section{Experiment III: Exploratory}

Further exploratory work was conducted at York University with Solvents 1 and 2 and a solvent system using methanol only. Four types of plates were evaluated: cellulose, silica gel, aluminum oxide, polyamide. Two-dimensional chromatography was used also with various combinations of solvents and TLC plates. The results were the same as in Experiment I; Solvent 1 with cellulose plates provided the best resolution of sample spots with one-dimensional chromatography.

\section{Experiment IV}

A single nonstimulated control rat and one rat sacrificed after 6 CC were used. Samples of the supernatant from these two were spotted on a cellulose plate and run twice in Solvent 1. Again
B
SF

$\Theta$

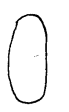

9

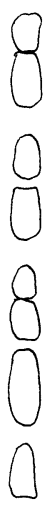

Q

0

Q

$\bigcap$

0

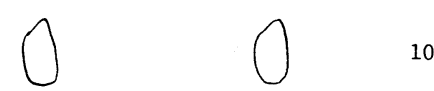

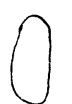

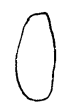

E

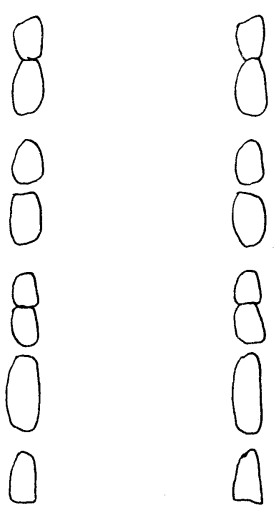

Figure 1. A drawing of the chromatographic patterns obtained with cellulose plates in Solvent 1 (n butanol-ethanc1acetic acid-water, 8:2:1:3). (A) The patterns in Experiments II, IV, and for the nonseparated samples and the fractions $>10,000$ $M W$ in Experiment V. (B) The patterns in Experiment $V$ for samples $<500$ and 500 to $10,000 \mathrm{MW}$. 0 , the origin, the point of application of samples; 1 to 10 , each of the 10 sample spots; $\mathrm{SF}$, solvent front; $\mathrm{E}$, rats stimulated to a specific point in the kindling process, usually to a number of $\mathrm{CC} ; \mathrm{C}$, nonstimulated controls.

10 spots emerged, as in Experiment II. Rf values were approximately the same as before.

\section{Experiment V}

In this final experiment, two nonstimulated controls and two rats after $6 \mathrm{CC}$ were employed. Approximately one-third of the volume of $\mathrm{E}$ and $\mathrm{C}$ supernatants was used, as in the previous experiments. The remaining materials from $\mathrm{E}$ and $\mathrm{C}$ were run separately in an Amicon ultrafiltration cell using UM 05 and UM 10 membranes to separate the samples into three fractions according to molecular weight (MW): $<500$, 500$10,000,>10,000$. Thus, there were four samples for $\mathrm{E}$ and four for $C$. Each of the samples was spotted on a cellulose plate and run in Solvent 1. There were two separate runs. There were no differences between $\mathrm{E}$ and $C$ in each of the four portions (Figure 1). In the 
nonseparated fraction and that whose MW was greater than 10,000, there were 10 spots, as in Experiments II and IV (Figure 1A). In the other fractions, there were only nine spots; the fastest moving spot was missing (Figure 1B). The $\mathrm{Rf}$ values were approximately the same as before.

\section{DISCUSSION}

The results indicate quite clearly that there are no chemical changes during the kindling process which are detectable by TLC with the solvent systems and plates which we used. The pattern for $\mathrm{E}$ and $\mathrm{C}$ rats at different stages was the same. The only difference detected was for samples less than $10,000 \mathrm{MW}$ and those containing materials of greater molecular weight. This difference probably was based on the presence of hemoglobin components in the fractions of $\mathrm{MW}>10,000$, for hemoglobin has a molecular weight well above this point. Consistent with this interpretation is the fact that samples of greater than $10,000 \mathrm{MW}$ had a slightly reddish (blood) color, whereas the other fractions were colorless. Although each brain is rinsed in physiological saline when it is extracted to remove excess blood, some blood always remains in the brain.

The purpose of the study was to attempt to determine if qualitative differences in amino acid constituents of polypeptides were present between $\mathrm{E}$ and $\mathrm{C}$ in the kindling process. Such a difference, especially in the fraction between a MW of 500 and 10,000 , would have been consistent with the results indicating a negative transfer effect in our previous studies. However, it is entirely possible that quantitative differences are responsible for the retardation effect. If so,
TLC would not indicate this aspect. Amino acid analyses would be required to detect quantitative differences.

On the other hand, it is possible that qualitative changes are occurring, not in polypeptides or protein components, but in some other chemicals. Finally, it is possible also that qualitative changes occur in polypeptides but were not in sufficient quantity to be detected by our procedures.

\section{REFERENCES}

GaIto, J. DNA complex and adaptive behavior. Englewood Cliffs, N.J: Prentice-Hall, 1971.

GaITo, J. Further results obtained with pairing of the "kindling effect" and the "transfer experiment." Physiological Psychology, $1975,3,237.239$.

GaIto, J. Pairing of the transfer experiment with the kindling paradigm: A summary of results. Bulletin of the Psychonomic Society, 1976, 7, 50-52.

GaIto, J., \& GaITo, S. T. Interanimal negative transfer of the kindling effect. Physiological Psychology, 1974, 2, 379-382.

Gaito, J., Hopkins, R. W., \& Pelletier, W. Interanimal transfer and chemical events underlying the kindling effect. Bulletin of the Psychonomic Society, 1973, 1, 319-321.

Goddard, G. V., McIntyre, D. C., \& Leech, C. K. A permanent change in brain function resulting from daily electrical stimulation. Experimental Neurology, 1969, 25, 295-330.

Ungar, G., Desiderio, D. M., \& Parr, W. Isolation, identification and synthesis of a specific-behavior-inducing brain peptide. Nature, 1972, 238, 198-202.

(Received for publication November 3, 1975.) 